Journal of Applied Mathematics and Stochastic Analysis, 16:2 (2003), 121-126.

Printed in the USA (C)2003 by North Atlantic Science Publishing Company

\title{
COMPLETE CONVERGENCE FOR NEGATIVELY DEPENDENT RANDOM VARIABLES
}

\author{
M. AMINI D. \\ Sistan and Baluchestan University, Department of Mathematics \\ Faculty of Science, Zahedan, Iran \\ E-mail: amini@hamoon.usb.ac.ir \\ and
}

A. BOZORGNIA

Ferdowski University, Department of Statistics

Faculty of Mathematical Science, Mashhad, Iran

E-mail: bozorg@math.um.ac.ir

(Received February 2001; Revised January 2003)

\begin{abstract}
In this paper, we study the complete convergence for the means $\frac{1}{n} \sum_{i=1}^{n} X_{i}$ and $\frac{1}{n^{\alpha}} \sum_{k=1}^{n} X_{n k}$ via. exponential bounds, where $\alpha>0$ and $\left\{X_{n}, n \geq 1\right\}$ is a sequence of negatively dependent random variables and $\left\{X_{n k}, 1 \leq k \leq n, n \geq 1\right\}$ is an array of rowwise pairwise negatively dependent random variables.

Key words: Complete Convergence, Negatively Dependent.
\end{abstract}

AMS (MOS) subject classification: 60E15, 60F15.

\section{Introduction}

Let $\left\{X_{n}, n \geq 1\right\}$ be a sequence of i.i.d., real random variables. Hsu and Rabbins [5] proved that if $E(X)=0$ and $E\left(X^{2}\right)<\infty$, then the sequence $\frac{1}{n} \sum_{i=1}^{n} X_{i}$ converges to 0 completely. (i.e., the series $\sum_{n=1}^{\infty} P\left[\left|S_{n}\right|>n \varepsilon\right]<\infty$, converges for every $\varepsilon>0$ ). Now let $\left\{X_{n}, n \geq 1\right\}$ be a sequence of negatively dependent real random variables. In this paper, we proved the complete convergence of the sequence $\frac{1}{n} \sum_{i=1}^{n} X_{i}$, via. exponential bounds. In addition if $\left\{X_{n k}, 1 \leq k \leq n, n \geq 1\right\}$ is an array of rowwise pairwise negatively dependent random variables, we proved complete convergence of the sequence $\left\{\frac{1}{n^{\alpha}} \sum_{k=1}^{n} X_{n k}, n \geq 1\right\}$ where $\alpha>0$. To prove these theorems we need to the following definitions and lemmas.

Definition 1: The random variables $X_{1}, \cdots, X_{n}$ are pairwise negatively dependent if

$$
P\left(X_{i} \leq x_{i}, X_{j} \leq x_{j}\right) \leq P\left(X_{i} \leq x_{i}\right) P\left(X_{j} \leq x_{j}\right),
$$


for all $x_{i}, x_{j} \in I R, i \neq j$. It can be shown that (1.1) is equivalent to

$$
P\left(X_{i}>x_{i}, X_{j}>x_{j}\right) \leq P\left(X_{i}>x_{i}\right) P\left(X_{j}>x_{j}\right),
$$

for all $x_{j}, x_{i} \in I R, i \neq j$.

Definition 2: The random variables $X_{1}, \cdots, X_{n}$ are said to be negatively dependent (ND) if we have

$$
P\left(\cap_{j=1}^{n}\left(X_{j} \leq x_{j}\right)\right) \leq \prod_{j=1}^{n} P\left(X_{j} \leq x_{j}\right),
$$

and

$$
P\left(\cap_{j=1}^{n}\left(X_{j}>x_{j}\right)\right) \leq \prod_{j=1}^{n} P\left(X_{j}>x_{j}\right),
$$

for all $x_{1}, \cdots, x_{n} \in I R$. An infinite sequence $\left\{X_{n}, n \geq 1\right\}$ is said to be ND if every finite subset $\left\{X_{1}, \cdots, X_{n}\right\}$ is ND.

Conditions (1.3) and (1.4) are equivalent for $n=2$. However Ebrahimi and Ghosh [4] show that these definitions do not agree for $n \geq 3$.

Definition 3: The sequence $\left\{X_{n}, n \geq 1\right\}$ of random variables converges to zero completely (denoted $\lim _{n \rightarrow \infty} X_{n}=0$ completely), if for every $\varepsilon>0$

$$
\sum_{n=1}^{\infty} P\left[\left|X_{n}\right|>\varepsilon\right]<\infty
$$

Lemma 1: (Petrov [8]) Let $X$ be a random variable with $E(X)=0, E\left(X^{2}\right)<\infty$, and suppose there exists a positive constant $H$ such that for all $m \geq 2$

$$
\left|E\left(X^{m}\right)\right| \leq \frac{1}{2} m ! \sigma^{2} H^{m-2}
$$

then for every $|t| \leq \frac{1}{2 H}$

$$
E e^{t X} \leq e^{t^{2} \sigma^{2}} .
$$

Lemma 2: (Serfeling [9]) Let $X$ be a r.v. with $E(X)=\mu$. If $P[a \leq X \leq b]=$ 1. Then for every real number $h>0$,

$$
E e^{h(X-\mu)} \leq e^{\frac{h^{2}(b-a)^{2}}{8}},
$$

and

$$
E e^{h|X-\mu|} \leq 2 e^{\frac{h^{2}(b-a)^{2}}{8}} .
$$

The next three lemmas will be needed in the proofs of the strong law of large numbers in the next section [3].

Lemma 3: Let $X_{1}, \cdots, X_{n}$ be $N D$ random variables and $f_{1}, \cdots, f_{n}$ be a sequence of Borel functions which all are monotone increasing (or all are monotone decreasing), then $f_{1}\left(X_{1}\right), \cdots, f_{n}\left(X_{n}\right)$ are $N D$ random variables.

Lemma 4: Let $X_{1}, \cdots, X_{n}$ be pairwise $N D$ random variables, then

$$
E\left(X_{i} X_{j}\right) \leq E\left(X_{i}\right) E\left(X_{j}\right), \quad \forall i \neq j .
$$


Lemma 5: Let $X_{1}, \cdots, X_{n}$ be $N D$ nonnegative random variables, then

$$
E\left[\prod_{j=1}^{n} X_{j}\right] \leq \prod_{j=1}^{n} E\left[X_{j}\right] .
$$

\section{Exponential Bounds and Complete Convergence}

In this section, we obtained some exponential bounds for probability $P\left[\left|S_{n}\right|>x\right]$ for every $x>0$ using Lemmas 1 and 2, and then we proved the complete convergence of the sequence $\left\{\frac{1}{n} \sum_{i=1}^{n} X_{i}\right\}$. We shall consider a sequence of ND random variables $\left\{X_{n}, n \geq 1\right\}$, with zero means and finite variances. We put

$$
S_{n}=\sum_{k=1}^{n} X_{k}, \quad B_{n}=\sum_{k=1}^{n} \sigma_{k}^{2}
$$

Theorem 1: Let $\left\{X_{n}, n \geq 1\right\}$ be a sequence of $N D$ r.v.'s and suppose there exists a positive constant $H$ such that for all $m \geq 2$ and $1 \leq k \leq n$,

$$
\left|E\left(X_{k}^{m}\right)\right| \leq \frac{1}{2} m ! \sigma_{k}^{2} H^{m-2}
$$

if $\sum_{n=1}^{\infty} \exp \left[-\frac{n^{2} \varepsilon^{2}}{4 B_{n}}\right]<\infty$, for every $\varepsilon>0$, then

$$
\frac{1}{n} \sum_{k=1}^{n} X_{k} \longrightarrow 0, \quad \text { completely. }
$$

Proof: By Lemmas 1, 3, 5 and Markov's inequality for every $|t| \leq \frac{1}{2 H}$ we have

$$
\begin{aligned}
P\left[\left|S_{n}\right|\right. & \geq x] \leq P\left[S_{n} \geq x\right]+P\left[-S_{n} \geq x\right] \leq e^{-t x} E e^{t S_{n}}+e^{-t x} E e^{-t S_{n}} \\
& \leq e^{-t x}\left(\prod_{k=1}^{n} E e^{t X_{k}}+\prod_{k=1}^{n} E e^{-t X_{k}}\right) \leq 2 \exp \left[-t x+t^{2} B_{n}\right] .
\end{aligned}
$$

Hence

$$
P\left[\left|S_{n}\right| \geq x\right] \leq 2 \exp \left[-t x+t^{2} B_{n}\right] .
$$

With $h(t)=t^{2} B_{n}-t x$ and $0 \leq x \leq \frac{B_{n}}{H}$, the equation $h^{\prime}(t)=0$ has the unique solution $t=\frac{x}{2 B_{n}}$ which minimize $h(t)$. Hence

$$
P\left[\left|S_{n}\right| \geq x\right] \leq 2 \exp \left[-\frac{x^{2}}{4 B_{n}}\right] \quad \text { if } \quad 0 \leq x \leq \frac{B_{n}}{H}
$$

Let $a=\frac{B_{n^{\star}}}{H}$, where $n^{\star}$ is the first subscript so that $B_{n}>0$. Then for every $0<\varepsilon \leq a$, and by the assumption

$$
\sum_{n=1}^{\infty} P\left[\frac{\left|S_{n}\right|}{n} \geq \varepsilon\right] \leq \sum_{n=1}^{\infty} 2 \exp \left[-\frac{n^{2} \varepsilon^{2}}{4 B_{n}}\right]<\infty
$$


and for each $\varepsilon^{\prime}>a \geq \varepsilon>0$, we have

$$
\left[\sum_{n=1}^{\infty} P\left[\frac{\left|S_{n}\right|}{n} \geq \varepsilon^{\prime}\right] \leq \sum_{n=1}^{\infty} P\left[\frac{\left|S_{n}\right|}{n} \geq \varepsilon\right]<\infty .\right.
$$

These complete the proof.

Remark 1: In particular if $B_{n}=O\left(n^{\alpha}\right), 0<\alpha<2$, then series $\sum_{n=1}^{\infty} \exp \left[-\frac{n^{2} \varepsilon^{2}}{4 B_{n}}\right]$ converges.

Remark 2: If the random variables $X_{1}, X_{2}, \cdots, X_{n}$ are ND r.v.'s with zero means and uniformly bounded, that is if there exists a positive constant $c$ such that

$$
P\left[\left|X_{k}\right| \leq c\right]=1, \quad k \geq 1
$$

then for all integers $m \geq 2$ we have

$$
\left|E\left(X_{k}^{m}\right)\right| \leq c^{m-2} \sigma_{k}^{2}
$$

Thus Condition (1.6) in Lemma 1 is satisfied with $H=c$. Hence if $\sum_{n=1}^{\infty} \exp \left[-\frac{n^{2} \varepsilon^{2}}{4 B_{n}}\right]<$ $\infty$, for every $\varepsilon>0$, then

$$
\frac{1}{n} \sum_{k=1}^{n} X_{k} \longrightarrow 0, \quad \text { completely }
$$

Theorem 2: Let $\left\{X_{n}, n \geq 1\right\}$ be a sequence of $N D$ random variables and $c_{n}=\max \left\{\right.$ ess $\left.\sup \frac{\left|X_{k}\right|}{\sqrt{B_{n}}}, 1 \leq k \leq n\right\}$. If $\sum_{n=1}^{\infty} \exp \left[-\frac{2 n \varepsilon^{2}}{c_{n}^{2} B_{n}}\right]<\infty$, then for each $\varepsilon>0$,

$$
\frac{1}{n} \sum_{k=1}^{n} X_{k} \longrightarrow 0, \quad \text { completely. }
$$

Proof: By Lemmas 2, 3, 5 and Markov's inequality for every $t>0$, we have

$$
\begin{gathered}
P\left[\left|S_{n}\right|>\varepsilon\right] \leq P\left[S_{n}>\varepsilon\right]+P\left[-S_{n}>\varepsilon\right] \leq e^{-\frac{t \varepsilon}{\sqrt{B_{n}}}} E e^{\frac{t S_{n}}{\sqrt{B_{n}}}}+e^{-\frac{t \varepsilon}{\sqrt{B_{n}}}} E e^{\frac{-t S_{n}}{\sqrt{B_{n}}}} \\
\leq e^{-\frac{t \varepsilon}{\sqrt{B_{n}}}}\left\{\prod_{k=1}^{n}\left(E e^{\frac{t X_{k}}{\sqrt{B_{n}}}}+E e^{\frac{-t X_{k}}{\sqrt{B_{n}}}}\right)\right\} \leq 2 \exp \left[-\frac{t \varepsilon}{\sqrt{B_{n}}}+\frac{n t^{2} c_{n}^{2}}{2}\right] .
\end{gathered}
$$

Thus, for $t=\frac{\varepsilon}{n c_{n}^{2} \sqrt{B_{n}}}$ we have

$$
P\left[\left|S_{n}\right|>\varepsilon\right] \leq 2 \exp \left[-\frac{\varepsilon^{2}}{2 n c_{n}^{2} B_{n}}\right]
$$

and by the assumption we have

$$
\sum_{n=1}^{\infty} P\left[\frac{\left|S_{n}\right|}{n}>\varepsilon\right] \leq 2 \sum_{n=1}^{\infty} \exp \left[-\frac{n \varepsilon^{2}}{2 c_{n}^{2} B_{n}}\right]<\infty
$$

which completes the proof.

Remark 3: In particular if $c_{n}^{2} B_{n}=O\left(n^{\alpha}\right), 0<\alpha<1$, then series $\sum_{n=1}^{\infty} \exp \left[-\frac{n \varepsilon^{2}}{2 c_{n} B_{n}}\right]$ converges. 


\section{Strong Limit Theorem for arrays}

Let $\left\{X_{n k}, 1 \leq k \leq n, n \geq 1\right\}$ be an array of rowwise pairwise ND random variables with

$$
E\left[X_{n k}\right]=0, \quad \sigma_{n k}^{2}=E\left[X_{n k}^{2}\right], \quad 1 \leq k \leq n, n \geq 1 .
$$

We consider the means $\xi_{n}=\frac{1}{n^{\alpha}} \sum_{k=1}^{n} X_{n k}, n \geq 1$ where $\alpha$ is a fixed positive real number. Since $X_{n k}, 1 \leq k \leq n$ are pairwise ND random variables, by Lemma 4 we can write

$$
E\left[\xi_{n}^{2}\right] \leq \frac{1}{n^{2 \alpha}} \sum_{k=1}^{n} \sigma_{n k}^{2},
$$

because

$$
\begin{gathered}
E\left[\xi_{n}^{2}\right]=E\left[\frac{1}{n^{\alpha}} \sum_{k=1}^{n} X_{n k}\right]^{2}=\frac{1}{n^{2 \alpha}} \sum_{k=1}^{n} \sum_{j=1}^{n} E\left[X_{n k} X_{n j}\right] \\
=\frac{1}{n^{2 \alpha}}\left[\sum_{k=1}^{n} E\left[X_{n k}^{2}\right]+\sum \sum_{k \neq j} E\left[X_{n k} X_{n j}\right]\right] \leq \frac{1}{n^{2 \alpha}} \sum_{k=1}^{n} \sigma_{n k}^{2} .
\end{gathered}
$$

Theorem 3: Let $\left\{X_{n k}, 1 \leq k \leq n, n \geq 1\right\}$ be an array of rowwise pairwise $N D$ random variables with $E\left[X_{n k}\right]=0$. If for some $\alpha>0$

$$
\sum_{n=1}^{\infty} \frac{1}{n^{2 \alpha}} \sum_{k=1}^{n} \sigma_{n k}^{2}<\infty
$$

then

$$
\frac{1}{n^{\alpha}} \sum_{k=1}^{n} X_{n k} \longrightarrow 0 \quad \text { completely. }
$$

Proof: By Chebyshev's inequality and (3.9), we have

$$
P\left[\left|\xi_{n}\right|>\varepsilon\right] \leq \frac{1}{\varepsilon^{2}} E\left[\xi_{n}^{2}\right] \leq \frac{1}{\varepsilon^{2} n^{2 \alpha}} \sum_{k=1}^{n} \sigma_{n k}^{2} .
$$

Since for some $\alpha>0$

$$
\sum_{n=1}^{\infty} P\left[\left|\xi_{n}\right|>\varepsilon\right] \leq \sum_{n=1}^{\infty} \frac{1}{\varepsilon^{2} n^{2 \alpha}} \sum_{k=1}^{n} \sigma_{n k}^{2}<\infty,
$$

by Definition 3

$$
\frac{1}{n^{\alpha}} \sum_{k=1}^{n} X_{n k} \longrightarrow 0 \quad \text { completely. }
$$

Corollary 1: Under assumptions of Theorem 3, let $\sigma_{n k} \leq \sigma_{k k}, k \geq 1, n \geq$ $(k+1)$. If $\sum_{k=1}^{\infty} \frac{\sigma_{k k}^{2}}{k^{2 \alpha-1}}<\infty$ for some $\alpha>\frac{1}{2}$ then

$$
\frac{1}{n^{\alpha}} \sum_{k=1}^{n} X_{n k} \longrightarrow 0 \quad \text { completely. }
$$


Proof: We have

$$
\begin{gathered}
\sum_{n=1}^{\infty} E\left[\xi_{n}^{2}\right] \leq \sum_{n=1}^{\infty} \frac{1}{n^{2 \alpha}} \sum_{k=1}^{n} \sigma_{n k}^{2} \leq \sum_{n=1}^{\infty} \frac{1}{n^{2 \alpha}} \sum_{k=1}^{n} \sigma_{k k}^{2} \\
=\sum_{k=1}^{\infty} \sigma_{k k}^{2} \sum_{n=k}^{\infty} \frac{1}{n^{2 \alpha}}=O(1) \sum_{k=1}^{\infty} \frac{\sigma_{k k}^{2}}{k^{2 \alpha-1}},
\end{gathered}
$$

then

$$
\sum_{n=1}^{\infty} P\left[\left|\xi_{n}\right|>\varepsilon\right] \leq O(1) \sum_{k=1}^{\infty} \frac{\sigma_{k k}^{2}}{k^{2 \alpha-1}}<\infty,
$$

which completes the proof.

Remark 4: The weaker condition $\sum_{k=1}^{\infty} \frac{\sigma_{k k}^{2}}{k^{2 \alpha}}<\infty$, for every $\alpha>0$, implies only the complete convergence of subsequence $\left\{\xi_{2^{p}}, p=0,1,2, \ldots.\right\}$, since

$$
\begin{gathered}
\sum_{p=0}^{\infty} E\left[\xi_{2^{p}}^{2}\right] \leq \sum_{p=0}^{\infty} \frac{1}{2^{2 \alpha p}} \sum_{k=1}^{2^{p}} \sigma_{k k}^{2} \\
=\sum_{k=1}^{\infty} \sigma_{k k}^{2} \sum_{p: 2^{p} \geq k} \frac{1}{2^{2 \alpha p}}=O(1) \sum_{k=1}^{\infty} \frac{\sigma_{k k}^{2}}{k^{2 \alpha}} .
\end{gathered}
$$

\section{Acknowledgments}

The authors would like to thank the referee for his careful reading of the manuscript and for many valuable suggestions which improved the presentation of the paper.

\section{References}

[1] Amini, D.M. and Bozorgnia,A., Negatively dependent bounded random variables, probability inequalities and the strong law of large numbers, J. of Appl. Math. and Stoch. Anal. 13:3 (2000), 261-267.

[2] Amini, D.M., Azarnoosh,H.A., and Bozorgnia,A., The almost sure convergence of weighted sums of ND uniformly bounded random variables, J. of Sci. Islamic Republic of Iran 10:2 (1999), 112-116.

[3] Bozorgnia, A., Patterson, R.F, and Taylor, R.L., Limit theorems for dependent random variables, In: World Congress Nonl. Anal. '92 (ed. by V. Lakshmikantham), Walter de Gruyter Publ., Berlin (1996), 1639-1650.

[4] Ebrahimi, N. and Ghosh, M., Multivariate negative dependence, Commun. Stat. Theory. Math. 4 (1981), 307-337.

[5] Hsu, P.L. and Robbins, H., Complete convergence and the law of large numbers, Proc.Nat.Acad. Sci. 33 (1947), 25-31.

[6] Laha, R.G and Rohatgi, V.K., Probability Theory, John Wiley \& Sons, New York 1979.

[7] Loève, M., Probability Theory, Van Nostrand Reinhold Company 1963.

[8] Petrov, V.V., Limit Theorems of Probability Theory, Oxford, New York 1995.

[9] Serfling, R.J., Approximation Theorems of Mathematical Statistics, John Wiley \& Sons, New York 1980. 


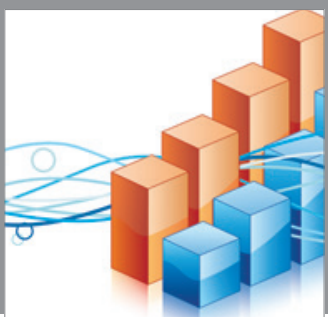

Advances in

Operations Research

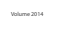

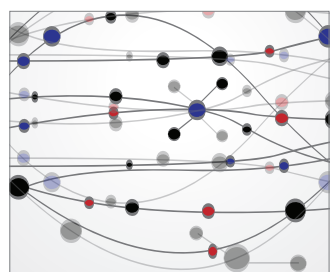

\section{The Scientific} World Journal
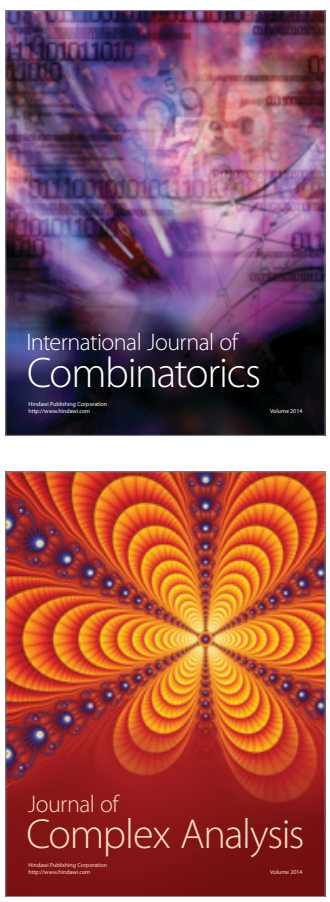

International Journal of

Mathematics and

Mathematical

Sciences
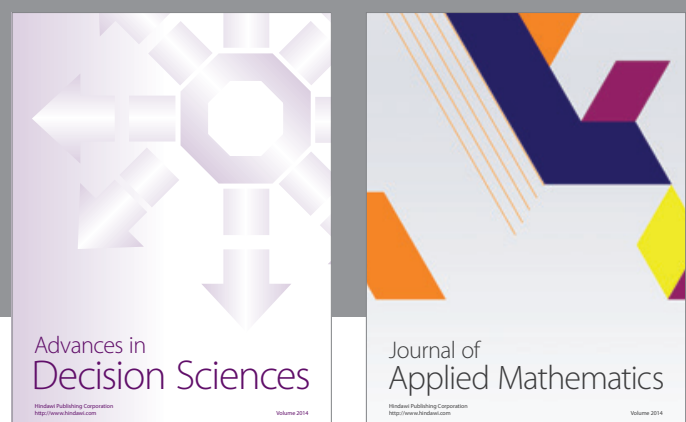

Journal of

Applied Mathematics
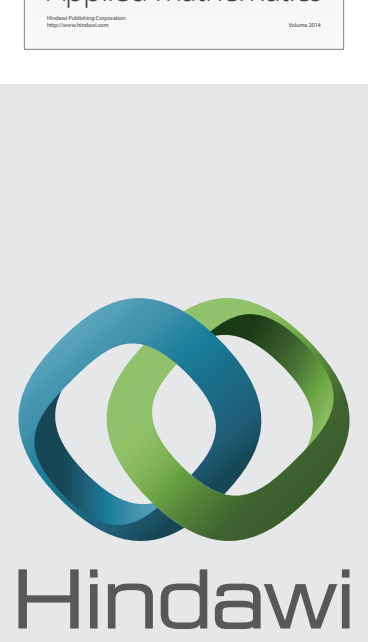

Submit your manuscripts at http://www.hindawi.com
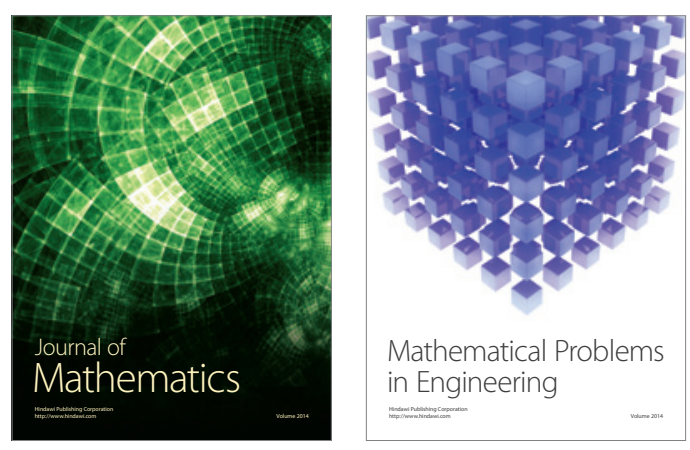

Mathematical Problems in Engineering
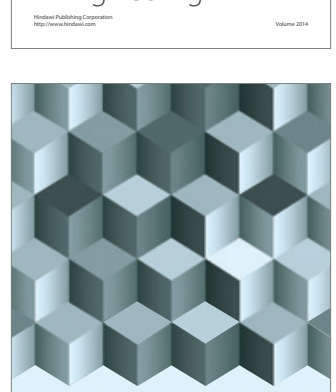

Journal of

Function Spaces
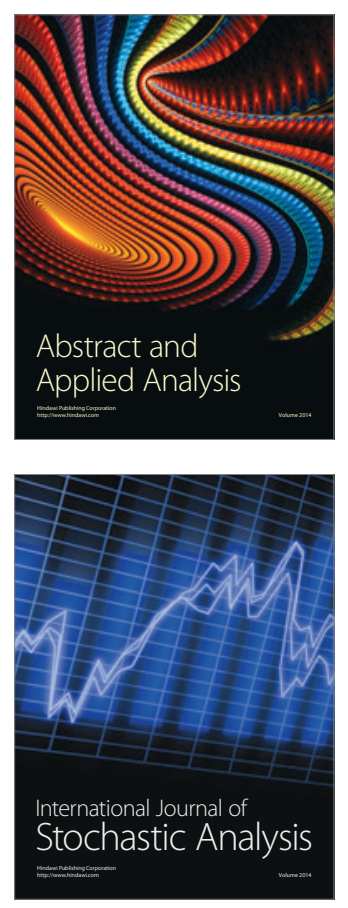

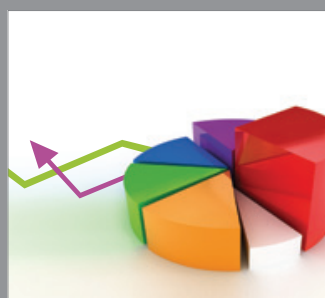

ournal of

Probability and Statistics

Promensencen
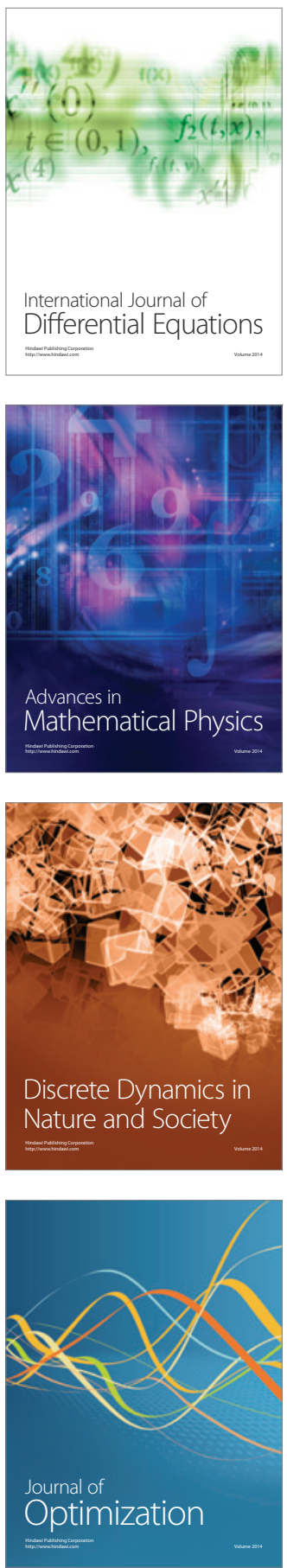